\title{
Magnetoledtherapy in the treatment of wounds after surgical procedures of the knee joint
}

\author{
This article was published in the following Dove Press journal: \\ Therapeutics and Clinical Risk Management \\ 2 September 2014 \\ Number of times this article has been viewed
}

Jarosław Pasek'

Tomasz Pasek ${ }^{2}$

Aleksander Sieroń

'Clinical Ward of Internal Diseases, Angiology, and Physical Medicine, Centre of Diagnostics and Laser Therapy in Bytom, Silesian Medical University, Katowice, Poland;

${ }^{2}$ Rehabilitation Unit, St Barbara Provincial Specialist Hospital No 5 , Sosnowiec, Poland
Correspondence: Jarosław Pasek Chair and Clinical Ward of Internal Diseases, Angiology, and Physical Medicine, Centre of Diagnostics and Laser Therapy in Bytom, Silesian Medical University, Katowice, ul Stepane Batoreop I5, 4I-902 Bytom, Poland

Tel +48327861630

Fax +48327861630

Email jarus_tomus@o2.pl

\begin{abstract}
The intense development of methods of physical medicine has been noted recently. The new methods are treatment methods, which in many cases allow a reduction of treatment time and positively influence the quality of life of patients undergoing treatment. This applies to illnesses and injuries of the locomotor system and diseases affecting soft tissues, as well as chronic wounds. This article discusses the positive results of the treatment of a 63-year-old woman with a persisting chronic wound of her right lower extremity after knee joint endoprosthesis surgery. The physical medicine method applied, in the form of magnetoledtherapy, contributed to complete wound healing and alleviation of pain suffered, as well as improvement of the quality of life of the treated patient.
\end{abstract}

Keywords: magnetoledtherapy, physical medicine, chronic wounds, tissue regeneration

\section{Introduction}

The treatment of wounds that are difficult to heal still poses a serious interdisciplinary medical problem. ${ }^{1}$ In many cases, such treatment requires patience and perseverance, from both the patient and the treating physician. In Poland, the problem of chronic wounds affects some 500,000 people a year, and those persons suffer for months not only because of the pain and the progressing disability but also because of social dysfunction. ${ }^{2}$ Despite substantial progress in discovering processes responsible for wound formation, the results of chronic wound treatment are still not completely satisfactory. Pharmacological treatment effectiveness varies. In many cases, the treatment is lengthy, far from convenient for the patient, and connected with a substantial level of discomfort. In addition, in a lot of cases, it fails to provide the expected results of therapy. In worst-case scenarios, chronic wounds may lead to generalized infections, disablement, or even death. ${ }^{1,3}$

Contemporary procedures applied in the case of wound treatment should include not only pharmacological treatment, surgical procedures, or proper dressing but also procedures belonging to physical medicine. ${ }^{3,4}$ In the local treatment of wounds, the proper selection of the treatment method is important and must be adequate to the phase of healing process, the degree of wound advancement phase, and the amount of exudate generated. That also applies to physical therapy methods, whose action is not only effective but also free from adverse effects. ${ }^{5,6}$

Recently, apart from classical means of treatment, which positively influence the wound healing process, new methods have been introduced in which physical factors are used..$^{6-8}$ Among those methods, there is magnetoledtherapy. Magnetoledtherapy is a joint application of extremely low frequency variable magnetic field applied, together with nonlaser optical radiation (ledtherapy), by means of innovative magnetic and light applicators. ${ }^{9,10}$ The mechanism behind the therapeutic influence in wound treatment 
consists of stimulating the regeneration of damaged blood vessels that serve the purpose of microcirculation, improvement of local blood supply in tissues, and improvement of blood rheological properties; increase of collagen production in fibroblasts; and acceleration of mitosis in the cells of epidermis germinative layer (stimulation of epidermization). Even during the first procedures one could notice the intensification of wound cleaning by the removal of necrotic tissues and inhibition of purulent discharge spots, which were gradually covered with fresh epidermis. ${ }^{6,9,11-13}$

\section{Aim of the study}

The aim of the study has been to present the beneficial results of treatment of a 63-year-old woman, with a persisting wound in her right lower limb after surgical endoplasty of the knee joint, who underwent magnetoledtherapy procedures with good effect.

\section{Case description}

The patient, (63 years of age), was admitted to the Chair and Clinic of Internal Medicine, Angiology and Physical Medicine and the Centre of Diagnostics and Laser Therapy of the Silesian Medical University in Katowice, Poland, because of a chronic wound, persisting for 4 months, in the area of the knee, resulting from a surgical wound occurring after endoplasty of the knee joint. Before admission to the clinic, the patient had undergone 3 months of outpatient treatment without satisfactory results.

On admission, the patient was found to have a wound in a persistent inflammatory condition and with pus secretion (Figure 1). Also on admission, in addition to the

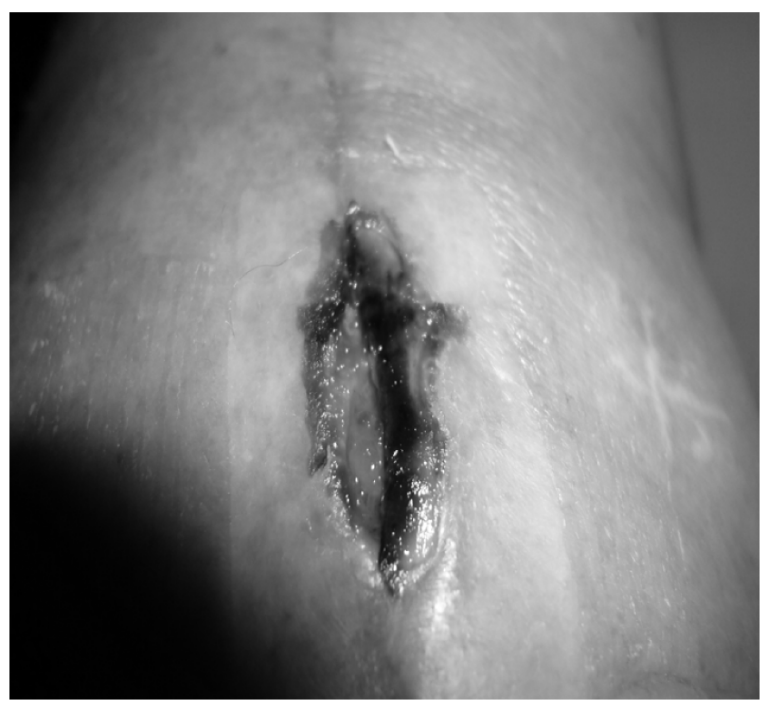

Figure I Photograph taken before starting the therapy. examination, the patient had surgical and angiologic consultations. The consulting surgeon cleaned the wound in admission room conditions. After that, physical treatment of the patient was initiated.

\section{Physical treatment}

The patient was subjected to magnetoledtherapy procedures, using a physical therapy device (Viofor JPS Standard) (Figure 2) with mixed red optic radiation (R)-infrared radiation (IR) panel applicators. The application was at the wound area, placing the applicators some $2-3 \mathrm{~cm}$ away from the surface to be exposed to radiation (the procedure was performed with the patient sitting). The procedures were performed in two series of 15 procedures each, with weekend breaks. The time distance between the two series of procedures was 4 weeks.

The luminous energy was produced by 280 specially selected high-energy light-emitting diodes emitting light waves and energy that provide most beneficial therapeutic results. The device functioned in the Magnetic and Light mode, emitting a variable magnetic field with $\mathrm{R}$ (wavelength, $630 \mathrm{~nm}$ ), as well as IR (wavelength, $850 \mathrm{~nm}$ ), for 12 minutes to the wound area, with a constant frequency of $181.8 \mathrm{~Hz}$ and therapeutic program M2P2.

M2 - application with increasing magnetic field intensity (magnetic field intensity increases every 10 or 12 seconds, to a pre-selected level, in cycles during the application procedure).

P2 - JPS system with two types of impulses, having the frequency of 180-195 Hz.

The magnetic field intensity amounted to 6 . The procedures were performed in outpatient mode, two times a day, daily, for 3 weeks. After each procedure, the wound was dressed in a protective dressing.

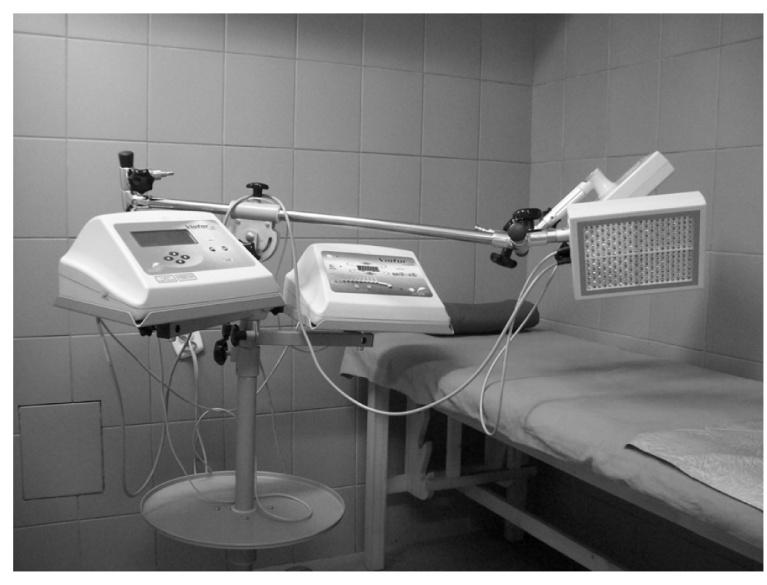

Figure 2 Apparat Viofor JPS Standard to magnetoledtherapy procedures. 


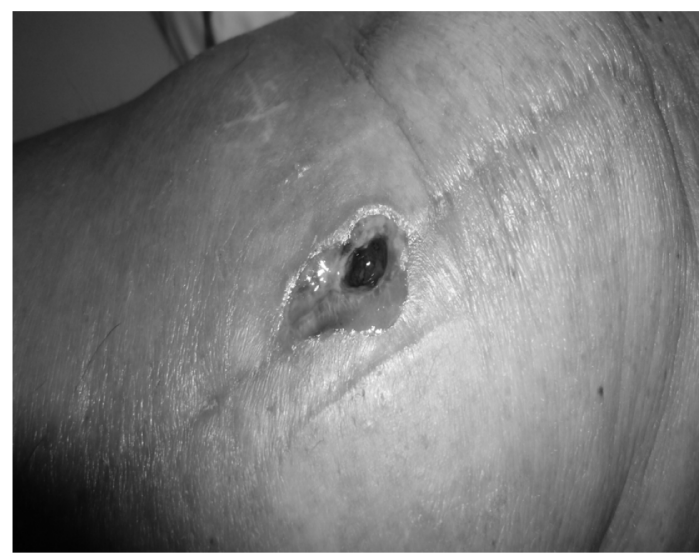

Figure 3 Photograph taken 15 days after beginning therapy.

\section{Results}

During the course of the first therapeutic session, the patient reported significant reduction of suffering caused by pain. In addition, a substantial reduction of edema and reddening around the wound was noticed. After the fourth procedure, the patient reported feeling "wound itching." After eight procedures ( 1.5 weeks after the therapy had begun), a photograph of the wound was taken (Figure 3 ).

The wound revealed reduction of inflammatory process and purulent discharge, as well as positive signs of accelerated healing, with the visible development of granulation tissue. In addition, the wound margin developed fine-grained pink granulation tissue, which gradually began to cover the wound with fresh epidermis. The very weak currents, which formed during interaction with piezoelectric substances, stimulated the activity of cells, which in turn promoted vasodilatation (direct influence on relaxation of smooth muscular coat of vessels, with substantial stimulation of neo-angiogenesis). Increased blood supply for tissues influenced their better oxygen supply positively, which evidently stimulated the processes of reconstruction and healing of damaged structures. In addition, a substantial reduction in pain intensity has been noticed.

After the full therapeutic cycle had been completed (30 procedures), complete healing of the surgical wound has been achieved. The final result of the therapy performed is presented in Figure 4.

After the treatment was completed, significant improvement of clinical condition was obtained in that patient, as well as improvement of the patient's mental condition, as the wound has visibly healed.

\section{Discussion}

The results obtained indicate it is proper to apply physical medicine procedures after orthopedic procedures. The obtained results have been confirmed in the studies done by Chaves et al and Borg et al. ${ }^{14,15}$ The beneficial effect of applying magnetic fields and low-energy light in wound treatment results from their biological effects, among which one can enumerate the intensification of oxygen utilization processes and tissue respiration, increased intensity of anaerobic respiration, processes in ischemic tissues, and vasodilatation and angiogenic activities, which cause the development of collateral circulation in the area where the wound forms..$^{13,16}$ In addition, those factors intensify regeneration processes (the stimulation of DNA replication processes, as well as synthesis of proteins and collagen) and repair, stimulating the epidermization process (acceleration of mitosis in cells of the germinative layer of epidermis) and inhibiting infection processes. ${ }^{17,18}$ The mechanism of therapeutic action of magnetic fields in such cases consists of stimulation of the regeneration of a destroyed network of vessels responsible for microcirculation, as well as enhancing of local blood supply
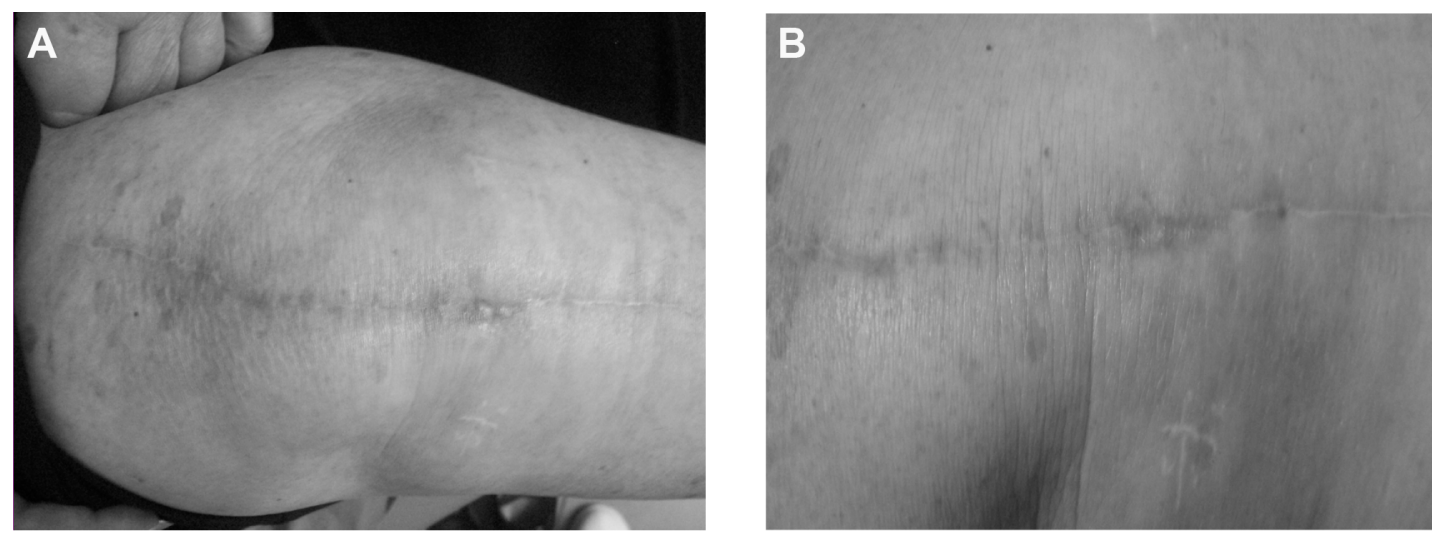

Figure 4 (A) Photograph taken at control visit in clinic after 3 months, at the end of therapy. (B) Photograph taken at control visit in clinic (after 3 months at the end of therapy). 
for tissues. Increased oxygen uptake is related to stimulation of tissue respiration processes, synthesis of DNA, and acceleration of mitotic cycle. ${ }^{6,19}$

\section{Wrap-up}

In medicine, physical treatment (heliotherapy, phototherapy, and balneotherapy) ranks among the oldest and most naturebased methods of treatment. ${ }^{15}$ The treatment efficiency of these methods depends largely on the type of pathological state to be treated, lesion intensity, general condition of the patient, and mainly, conscientious and strict obeying of the therapist's suggestions.

In the treatment of many diseases and conditions, a multidirectional treatment model should always be assumed. ${ }^{3}$ The chronic wounds that form (whatever their etiology) require cleaning by removal of necrotic tissue and purulent secretion in the exudative phase, whereas during granulation, tissue growth, and epidermization, stimulation of those processes is necessary. ${ }^{1,4}$ To achieve these aims in conservative treatment, application of contemporary physical methods proves to be among the most effective ways. ${ }^{19,20}$ Because of their biological activity, those methods stimulate the regeneration and repair processes, being a precious supplement to regular treatment.

Therapeutic results achieved through the application of magnetoledtherapy may be highly satisfactory, as they are connected with fast activity (analgesic and anti-inflammatory effect), without physical contact and without pain, which is very significant for the patient. The search for various solutions, which may bring about positive results of treatment, is a substantial clinical challenge, which in turn requires considerable knowledge and skills in planning of treatment.

\section{Conclusion}

The application of magnetoledtherapy procedures resulted in complete healing of the surgical wound that occurred after a knee arthroplasty procedure. In addition, multidirectional therapeutic management, which included physical medicine methods, resulted in positive effects in the form of wound healing and improved the quality of life of the patient.

\section{Disclosure}

The authors report no conflicts of interest in this work.

\section{References}

1. Vovden P, Apelqvist J, Moffat Ch. Wound Complexity and Healing. Hard-to-heal wounds: a holistic approach. 2008.

2. Sieroń A, Cierpka L, Rybak Z, Stanek A. Angiology textbook. AlfaMedica Press, Bielsko-Biała; 2009.

3. Schindzielorz M, Schmidt M. Einsatz neuer Konzepte im Wundmanagement. Sektorenübergreifende Wundversorgung [Implementing new concepts in wound management. Interdisciplinary wound management]. Pflege Z. 2011;64(3):158-160. German.

4. Caldwell MD. Wound surgery. Surg Clin North Am. 2010;90(6): $1125-1132$.

5. Taradaj J, Franek A, Dolibog P, Cierpka L, Błaszczak E. The physical treatment of chronic wound healing. Practical Rehab. 2008;1:34-35.

6. Carruthers J, Carruthers A. Commentary: electromagnetic radiation and wound healing. Dermatol Surg. 2012;38(3):451-453.

7. Costin GE, Birlea SA, Norris DA. Trends in wound repair: cellular and molecular basis of regenerative therapy using electromagnetic fields. Curr Mol Med. 2012;12(1):14-26.

8. Pasek J, Pasek T, Sieron A. Magnetostimulation in the treatment of crural vein ulceration. Int Wound J. 2012;9(5):566-569.

9. Sieron A, Pasek J, Mucha R. Magnetic fields and light energy in medicine and rehabilitation - magnetoledtherapy. Bal Pol. 2007;1(107):1-7.

10. Pasek J, Sieroń A. Ledtherapy. Practical Rehab. 2011;13:52-55.

11. Czyz CN, Foster JA, Lam VB, et al. Efficacy of pulsed electromagnetic energy in postoperative recovery from blepharoplasty. Dermatol Surg. 2012;38(3):445-450.

12. Pasek J, Mucha R, Sieron A. Crural ulceration: the treatment of low frequency magnetic field stimulation with high energetic diode LED. Case description. Acta Bio Optica Informatica Med. 2006;1(12):15-19.

13. Sieron A, Pasek J. Magnetic fields in the treatment of wounds. Practical Rehab. 2011;4:48-51.

14. Chaves ME, Araújo AR, Santos SF, Pinotti M, Oliveira LS. LED phototherapy improves healing of nipple trauma: a pilot study. Photomed Laser Surg. 2012;30(3):172-178.

15. Borg MJ, Marcuccio F, Poerio AM, Vangone A. Campi magnetici in terapia fisica. Esperienze in riabilitazione ortopedica e traumatologica. [Magnetic fields in physical therapy. Experience in orthopedics and traumatology rehabilitation]. Minerva Med. 1996;87(10):495-497. Italian.

16. Sieroń A, Pasek J, Mucha R. Low energy light in medicine and rehabilitation. Practical Rehab. 2007;1:25-27.

17. Oliveira Sampaio SC, de C Monteiro JS, Cangussú MC, et al. Effect of laser and LED phototherapies on the healing of cutaneous wound on healthy and iron-deficient Wistar rats and their impact on fibroblastic activity during wound healing. Lasers Med Sci. 2013;28(3):799-806.

18. Sieroń A, et al. The Use of Magnetic Fields in Medicine. 2nd ed. Bielsko-Biała, Poland; Alfa-Medica Press; 2002.

19. Annaratone G, Rapelli G, Graziano G. [Magnetotherapy in clinical and ambulatory practice]. Minerva Med. 1983;74(14-15):823-833.

20. Pasek J, Pasek T, Sieroń A. Home therapy with use a variable magnetic fields and light. Practical Rehab. 2007;3:50-53.
Therapeutics and Clinical Risk Management

\section{Publish your work in this journal}

Therapeutics and Clinical Risk Management is an international, peerreviewed journal of clinical therapeutics and risk management, focusing on concise rapid reporting of clinical studies in all therapeutic areas, outcomes, safety, and programs for the effective, safe, and sustained use of medicines. This journal is indexed on PubMed Central, CAS,

\section{Dovepress}

EMBase, Scopus and the Elsevier Bibliographic databases. The manuscript management system is completely online and includes a very quick and fair peer-review system, which is all easy to use. Visit $\mathrm{http}: / /$ www.dovepress.com/testimonials.php to read real quotes from published authors. 